\title{
A Novel Nanocomposite Particle of Hydroxyapatite and Silk Fibroin: Biomimetic Synthesis and Its Biocompatibility
}

\author{
Lin Niu, ${ }^{1}$ Rui Zou, ${ }^{1}$ Qida Liu, ${ }^{2}$ Quanli Li, ${ }^{3}$ Xinmin Chen, ${ }^{4}$ and Zhiqing Chen ${ }^{4}$ \\ ${ }^{1}$ Stomatological Hospital, Xi'an Jiaotong University, Xi'an 710004, China \\ ${ }^{2}$ SV Laboratory, School of Aerospace, Xi'an Jiaotong University, Xi'an 710049, China \\ ${ }^{3}$ Faculty of Stomatology, Anhui Mediical University, Hefei 230032, China \\ ${ }^{4}$ West China College of Stomatology, Sichuan University, Chengdu 610041, China \\ Correspondence should be addressed to Rui Zou, rainy@mail.xjtu.edu.cn
}

Received 12 February 2010; Revised 6 June 2010; Accepted 12 June 2010

Academic Editor: Gong Ru Lin

Copyright ( $) 2010$ Lin Niu et al. This is an open access article distributed under the Creative Commons Attribution License, which permits unrestricted use, distribution, and reproduction in any medium, provided the original work is properly cited.

A novel bone-like biomaterial of hydroxyapatite (HAP) and silk fibroin (SF) composite was developed by biomimetic synthesis. The composite was precipitated from drops of $\mathrm{Ca}(\mathrm{OH})_{2}$ suspension and $\mathrm{H}_{3} \mathrm{PO}_{4}$ solution with SF. With this method, the HAP nanocrystals were obtained by self-assembling on a SF surface whose c-axis was aligned with the long-axis direction of SF in microstructures; this shares the same misconstrues of collagen and HAP with that in the natural bone. The HAP/SF composite then demonstrated that it could promote osteoblast proliferation in vitro and new bone formation in vivo. The novel biomaterial is a promising material for bone replacement and regeneration.

\section{Introduction}

The Silkworm protein, composed of mainly sericin and fibroin proteins, is made naturally by a silk moth, Bombyx mori. While the Sericin protein is immunological, purified silk fibroin (SF), a linear polypeptide with $\beta$-sheet structure, exhibits little or no immunological reactions and retains many of the desirable features of silk material [1]. Due to its excellent features of biological compatibility, mechanical strength, and biodegradation, SF can be useful as an engineering biomaterial in a number of biomedical and biotechnological applications [2-5].

Various types of template material for fabricating bonelike composite have been reported. Some organic templates could be collagen, SF, chitosan, or chondroitin sulfate [6-10]. However, SF has the unique feature of being a novel organic template due to its excellent biological compatibility and mechanical strength. According to the literature, the HAP/SF composite can be prepared using biomimetic mineralization [11], mechanochemical route [12], or coprecipitation methods $[8,13]$. The composite obtained by these methods shares the similar features with natural bone in structure and composition; however, few reports can be found in the literature on these microstructure features of composite in the nanometer level.

In this study, SF was used as an organic template obtaining for the HAP/SF composite biomaterial, while biomimetic synthesis is for the bone-like feature in the HAP/SF composite. The nanostructure of HAP/SF composite is close to that in natural bone whose $\mathrm{c}$-axis of HAP crystal is paralleled with the long axis of organic template. The biocompatibility of HAP/SF was then evaluated in vitro and in vivo.

\section{Experiment}

2.1. Materials. Bombyx mori silks were purchased from Tianyou Silk Co, LTD (Sichuan, China). Analytical grade Natrium carbonicum, calcium chloride, ethanol, and other chemical reagents were obtained from Changzheng Chemical Agent Company (Chengdu, China). Bag filters made from acetyl cellulose with a molecular weight cutoff of 10,000 D was obtained commercially from Beijing Solarbio Science \& Technology Co. Ltd. (Beijing, China). Dulbecco's Modified Eagle's Medium (DMEM) and 10\% fetal 
bovine serum were from GIBCO Life Technologies (Grand Island, NY, USA). MTT [3-(4,5-dimethylthiazol-2-yl)-2,5diphenyltetra-zoliumbromide], dimethylsulfoxide (DMSO), penicillin, and streptomycin were purchased from SigmaAldrich Inc., USA.

2.2. Silk Fibroin Preparation. SF was prepared as previously described by Santin et al. [3] and H.-J. Jin et al. [4]. In order to remove sericin, the domestic (Bombyx mori) silks were degummed twice by sequentially boiling them in $0.5 \mathrm{wt} \%$ $\mathrm{Na}_{2} \mathrm{CO}_{3}$ solution for 30 minutes. Then, they were rinsed and air-dried. The pure SF were dissolved with a triad solution of calcium chloride, ethanol, and distilled water $(1: 2: 8$ mole ration) at $80^{\circ} \mathrm{C}$ using a homeothermia swing bed. The prepared solution was purified by dialyzed against distilled water for 3 days. The solution was then concentrated and lyophilized, to yield purified SF.

2.3. Fabrication of HAP/SF Composite. The HAP/SF composite was obtained by a $\mathrm{Ca}(\mathrm{OH})_{2}$ suspension and $\mathrm{H}_{3} \mathrm{PO}_{4}{ }^{-}$ SF mixed solution using biomimetic synthesis method. The theoretical mass proportion of the inorganic component to organic component was $7: 3$ according the ratio of natural bone. The $\mathrm{H}_{3} \mathrm{PO}_{4}$-SF solution was prepared by adding $21.8 \mathrm{ml}$ of $11.8 \mathrm{mg} / \mathrm{ml} \mathrm{SF}$ solution to $41.5 \mathrm{ml}$ of $0.087 \mathrm{M}$ $\mathrm{H}_{3} \mathrm{PO}_{4}$ solution and stirring vigorously. Subsequently, the prepared $\mathrm{H}_{3} \mathrm{PO}_{4}$-SF solution was slowly dropped into $300 \mathrm{ml}$ of $0.02 \mathrm{M} \mathrm{Ca}(\mathrm{OH})_{2}$ suspensions at the speed of $60 \mathrm{drops} / \mathrm{min}$ and applied by a vigorous stirring simultaneously. The whole preparation was in a water bath with a temperature of $70^{\circ} \mathrm{C}$, and the $\mathrm{pH}$ between 9 and 10 with $0.1 \mathrm{M} \mathrm{NaOH}$. During the process, the precipitate was observed within the solution; the supernatant was then decanted, and the precipitate was rinsed with distilled water. Finally, the precipitate powder was collected by vacuum dehydration.

The precipitate powder was characterized using Xray diffractometry (XRD) (X'Pert Pro MPD, Philips, Holland), Fourier-transform infrared (FTIR) spectrophotometry (Nicolet 510P FTIR, USA), scanning electron microscopy (SEM) (S-2460N, Hitachi, Tokyo, Japan) and transmission electron microscopy (TEM) (JEM-100CXII, Japan).

2.4. Cytocompatibility of HAP/SF Composite In Vitro. Human osteoblast-like cells (MG-63) were obtained from ATCC (Manassas, VA, USA). MG-63 cells were cultivated in Dulbecco's Modified Eagle's Medium supplemented with $10 \%$ fetal bovine serum at $37^{\circ} \mathrm{C}$ in a humidified atmosphere of $5 \% \mathrm{CO}_{2}$. The HAP/SF and HAP particle were fabricated into disc shapes (12 $\mathrm{mm}$ in diameter) by an FW3 compression machine (TianJin, China) and sterilized with epoxyethane. The cell suspension was seeded at a density of $1 \times 10^{4}$ cells $/ \mathrm{ml}$ into 6-well plates containing HAP/SF and HAP discs and cultured at the conditions described above.

The cells on the discs were cultured for 6 hours, 1 day, or 5 days and fixed overnight with a $3 \%$ glutaraldehyde solution in PBS at room temperature. After dehydration with $50 \%$, $60 \%, 75 \%, 85 \%, 95 \%$, and $100 \%$ alcohols for 15 minutes, substitution with isoamylacetate solution for 15 minutes, and

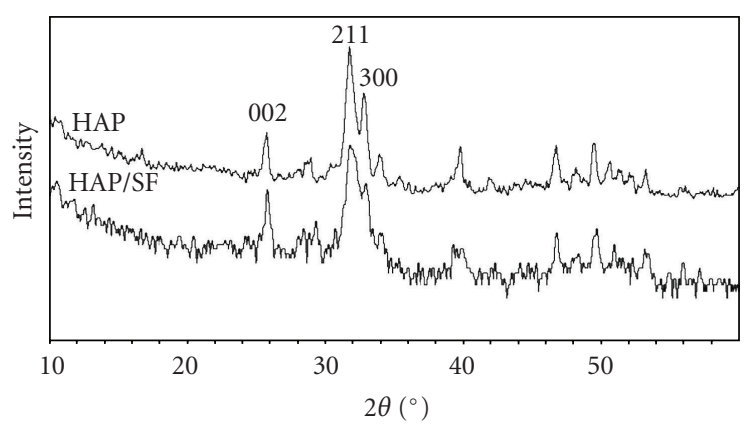

FIgURE 1: XRD spectra of HAP/SF and HAP.

critical point dry for 30 minutes, the samples were observed under SEM.

The viability of MG-63 cells was measured by MTT [3(4, 5-dimethylthiazol-2-yl)2,5-diphenyltetrazoliumbromide] assay on the 1st, $3 \mathrm{rd}, 5$ th, and 7 th days of culture. Absorbance was read on an HTS 7000 plus BioAssay Reader (PERKIN ELMER Ltd., Buckinghamshire, U.K.) at $570 \mathrm{~nm}$.

2.5. Tissue Response to HAP/SF Particle In Vivo. Twelve white New Zealand adult rabbits $(2.0-3.0 \mathrm{~kg})$ were used. They were anaesthetized by an injection of sodium pentobarbital $(24 \mathrm{mg} / \mathrm{kg}$ body weight) in the auricular vein. A hole of about $3-\mathrm{mm}$ in diameter was drilled in the mid-diaphysis of the femur, and the HAP/SF and HAP particle sterilized with epoxyethane was inserted into the hole. HAP served as the control group. Six animals were killed at 4 and 8 weeks postoperation. The bone fragments concluding the implants were harvested and the samples were fixed in a $10 \%$ buffered formalin solution, decalcified by a $10 \%$ EDTA solution, dehydrated in a graded ethanol series, and embedded in paraffin. Tissue blocks were sectioned and stained by hematoxylin and eosin (H\&E) for observation.

\section{Results and Discussion}

3.1. Characterization of HAP/SF Composite. Figure 1 shows the results of XRD measurement on the HAP/SF sample. There are three sharp peaks at 002,211 , and 300 characteristic of HAP around 25-35 . The observed three peaks were broader than pure standard HAP due to its low crystallinity of HAP in the HAP/SF composite. The crystal size of HAP is about $22 \mathrm{~nm}$ according to the XRD line.

FTIR spectra of HAP/SF and SF is shown in Figure 2 and denoted by the solid lines (a) and (b), respectively. The absorption bands of SF at 1650 and $1516 \mathrm{~cm}^{-1}$ are amide I and amide II $(\mathrm{N}-\mathrm{H})$, while the amide $\operatorname{III}(\mathrm{C}-\mathrm{N})$ of fibroin is at $1234 \mathrm{~cm}^{-1}$ (for details, see solid line b). Compared with SF, the amide I absorption HAP/SF is weakened obviously and the absorption band was shifted to $1659 \mathrm{~cm}^{-1}$ and the absorption of amide II and amide III also obviously weakened. The peaks of absorption bands at 1036, 962, 603, and $563 \mathrm{~cm}^{-1}$ in the HAP/SF composite is corresponding to the characteristic of $\mathrm{PO}_{4}{ }^{3-}$, while the peak characteristic of $\mathrm{OH}^{-}$is at $3567 \mathrm{~cm}^{-1}$. These differences suggest that there is 


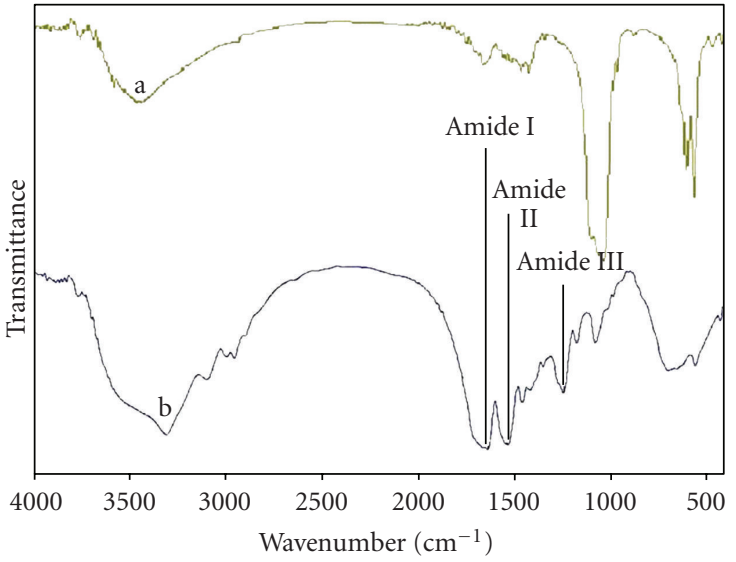

FIGURE 2: FTIR spectra of HAP/SF (a) and SF (b).

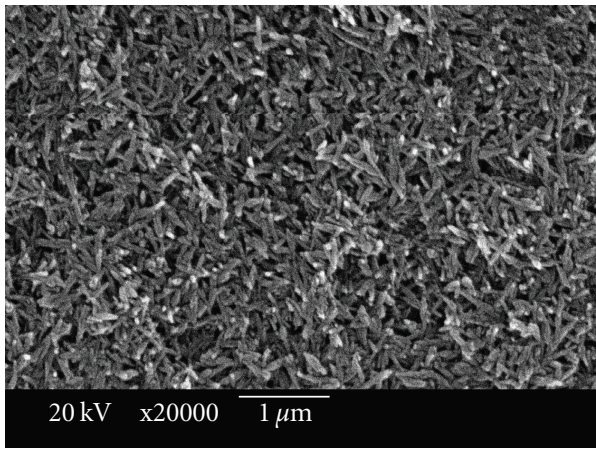

FIGURE 3: SEM microphotograph of the HAP/SF.

distinguished chemical interaction between the HAP crystal and SF. The peak at $1419 \mathrm{~cm}^{-1}$ can be distinguished by $\mathrm{CO}_{3}{ }^{2-}$, suggesting that carbonate ion was incorporated into the HAP crystal $[13,14]$. The characteristic coincides with that in natural bone.

The SF contains approximately $3 \%$ of acidic amino acids (Aspartate and Glutamate). Acidic amino acids containing many carboxyl $(-\mathrm{COOH})$ groups provide the active surface structure, which could initiate the HAP deposition and growth. The carboxyl groups, as an active group, play an effective role in apatite deposition and nucleation within the body environment. For this reason, the silk fibroin may act as a template to induce the deposition of apatite crystals. During the deposition, the calcium ions bind to the active carboxyl group and form complexes of $-\mathrm{COOCa}{ }^{+}$and $(-\mathrm{COO})_{2} \mathrm{Ca}$ on the surface of SF. Alkalinity solution promotes this action and facilitates apatite deposition and growth [11, 15-17].

As seen in Figure 3, the needle-like HAP/SF particles are randomly deposited in three dimensions. Each particle is about $400 \mathrm{~nm}$ in length. During the formation of the HAP/SF composite, SF act as an organic matrix to provide active sites for the nucleation and growth of HAP crystals [11]. Wang et al. [8] concluded that the SF can provide enough active sites and promote the self-assembly of HAP crystal on

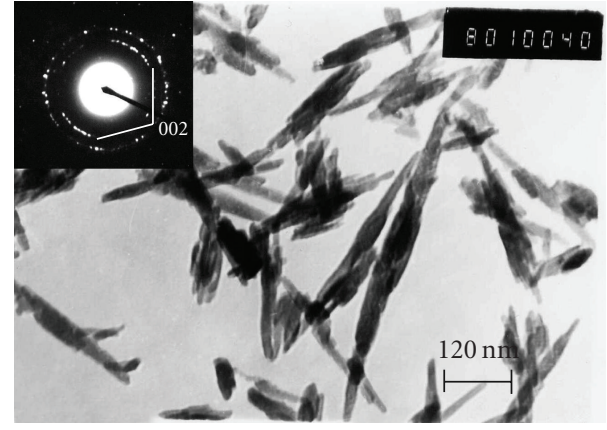

FIGURE 4: TEM of the HAP/SF composition and electron diffraction pattern $(\times 80000)$.

SF when the mass percentage of SF in HAP/SF composite achieves about $30 \%$. However, redundant quantities of SF may cause severe entanglement of the microstructure of HAP/SF. Therefore, the percentage of $30 \%$ of SF in HAP/SF is used in this paper.

TEM results showed that HAP nanocrystals were attached to the SF template (Figure 4). The size of HAP/SF particle is approximately $400 \times 60 \mathrm{~nm}$ and the shape of the particle is needle-like. Each particle consists of about $400 \mathrm{~nm}$ length SF attached with HAP nanocrystals about $25 \mathrm{~nm}$ in length. The diffraction pattern of the particle is seen in the left-top corner of Figure 4; the outer diffraction pattern forms a ring while the inner diffraction pattern divides into a pair of short arches. In Figure 4, the pattern of short arch diffraction was ascribed to 002 of HAP and the c-axis of HAP nanocrystals was in the long axis direction of the SF template $[7,10,18]$.

3.2. Cytocompatibility of HAP/SF Composite In Vitro. The cytocompatibility of HAP/SF was estimated by MG63 cell cultivation as is shown in Figure 5. The MG63 cells adhered to the HAP/SF discs and spread by pseudopodia after 6 hours (Figure 5(a)). They began to spread with polygonal morphology and extended some pseudopods to contact each other after one day (Figure 5(c)). After 5 days, the cells rapidly proliferated and grew in an aggregated, multilayered form (Figure 5(e)). On the HAP discs, the cells only adhered with round morphology after 6 hours (Figure 5(b)). After 1 day, the cells began to spread with polygonal morphology and extended to contact each other, and cell morphology was irregular (Figure 5(d)). After 5 days, the cells grew in aggregates (Figure 5(f)).

The MG-63 cell viability on the HAP/SF and HAP discs were both assessed by the MTT assay. The results are presented in Figure 6. The cells cultured on the HAP/SF discs showed higher viability compared with the HAP discs. Each group included six samples. The difference was statistically significant on days $1,3,5$, and $7(P<.05)$.

The novel composite of HAP/SF showed excellent biocompatibility in vitro. It suggested that the HAP/SF composite promotes osteoblast attachment and adhesion and enhances proliferation and differentiation. The cell attachment and adhesion at an early stage was probably 


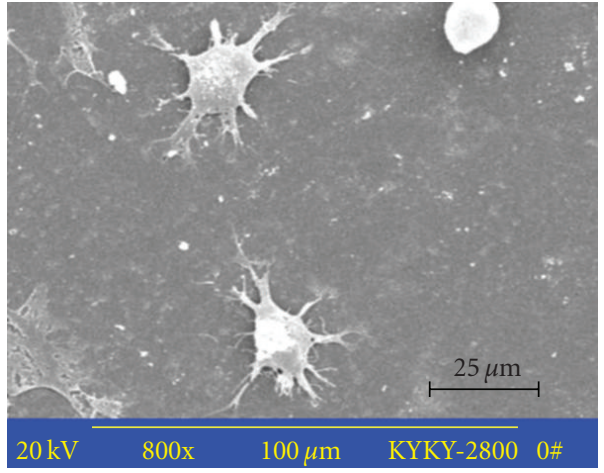

(a)

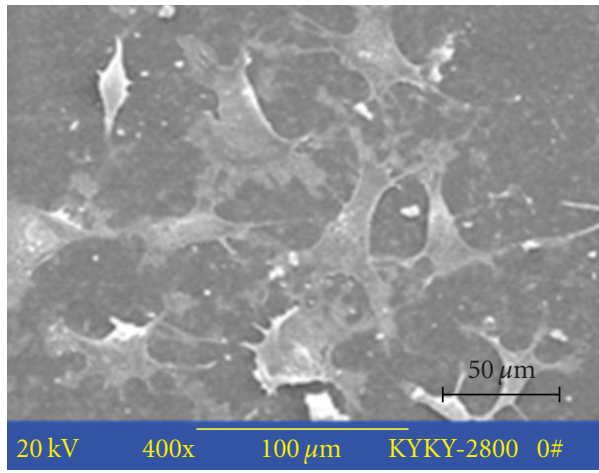

(c)

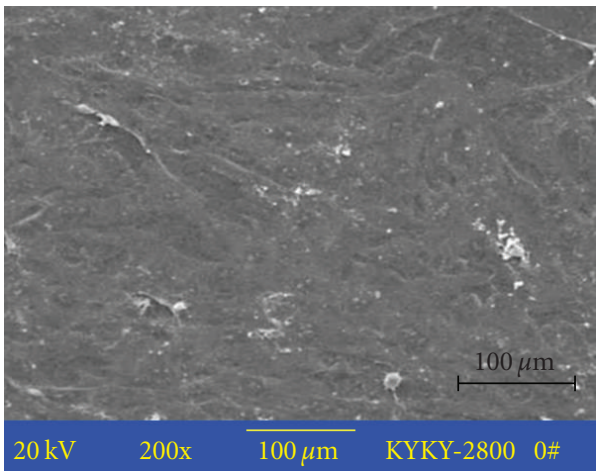

(e)

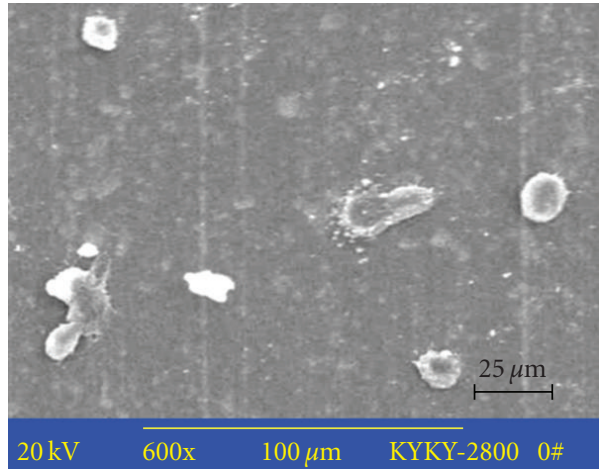

(b)

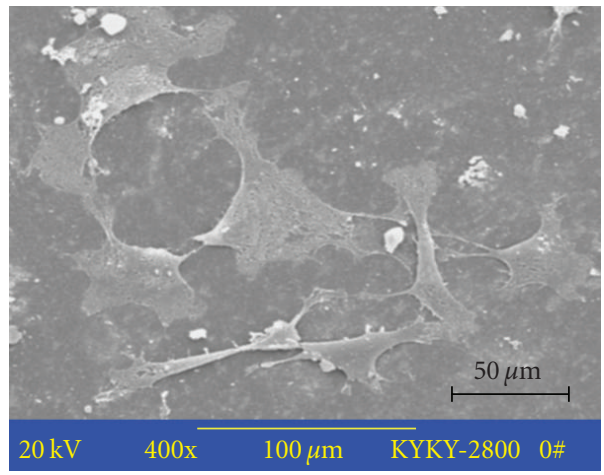

(d)

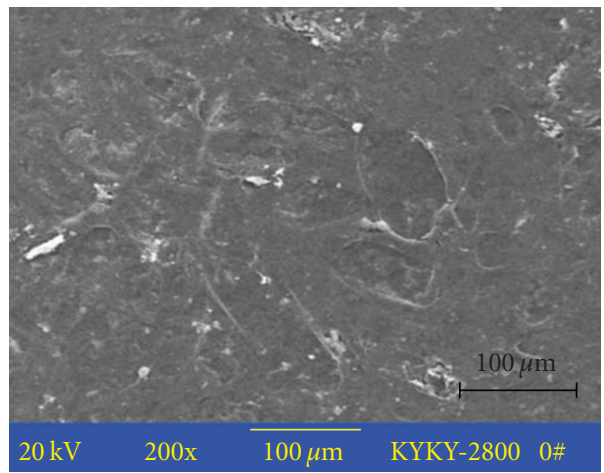

(f)

Figure 5: SEM of the cell culture on HAP/SF after $6 \mathrm{~h}$ (a), 1 day (c), 5 day (e), and HAP discs after $6 \mathrm{~h}$ (b), 1 day (d), 5 day (f).

a simple physicochemical process which can be determined by the roughness of the material surface. In subsequent cell migration, proliferation, and differentiation, extracellular matrix molecules (e.g., type I collagen) is required, for the cell function is adjusted by the molecules through the interaction with the cell-surface receptor of the integrin family [19-23].

Studies showed that SF can enhance the attachment, adhesion, and growth of cells and have the same efficiency with that of collagen $[24,25]$. This agrees with the results of this study. Furthermore, we also found that HAP/SF composite also promotes proliferation and differentiation. In the experiments, we observed that the MG-63 cells adhered to HAP/SF and spread by pseudopodia at 6 hours. The shape of MG-63 cells on HAP/SF display multilayer forms (see Figure 5(a)), while the round shape of MG-63 cells was found on HAP (see Figure 5(b)). It shows that cells on HAP/SF composite present excellent proliferation and differentiation.

3.3. Biocompatibility of HAP/SF Composite in Marrow Cavity. The biocompatibility of HAP/SF composite was conducted using the animal model of a rabbit. There are large numbers of HAP grains founded in marrow cavity of the rabbit at four weeks since operation, new bone tissue and marrow tissue were also found to grow into the interspaces of the grains in 


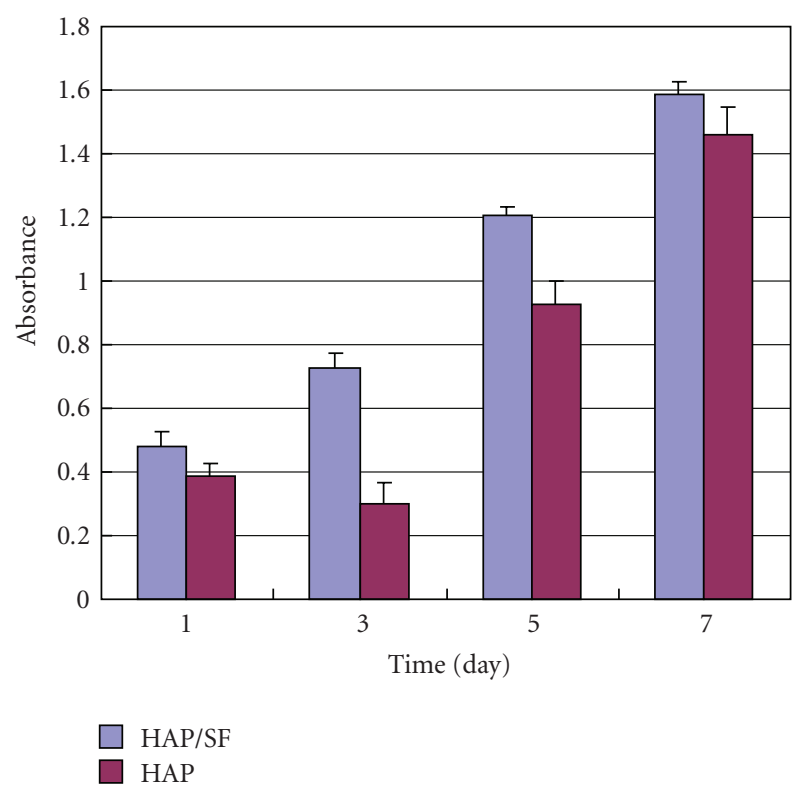

FIgURE 6: MTT test to evaluate the HAP/SF cytocompatibility ( $n=$ $6)$.

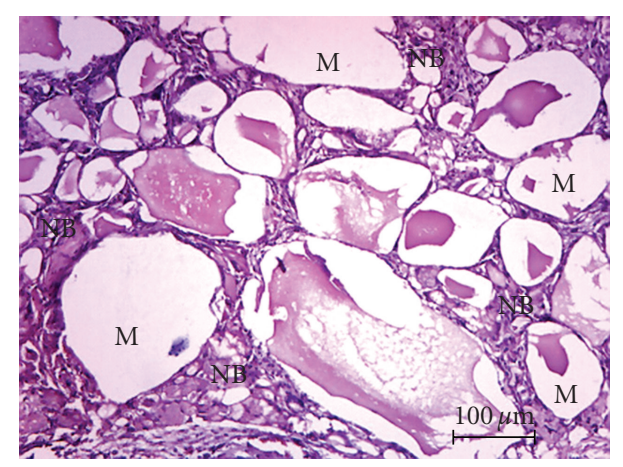

(a)

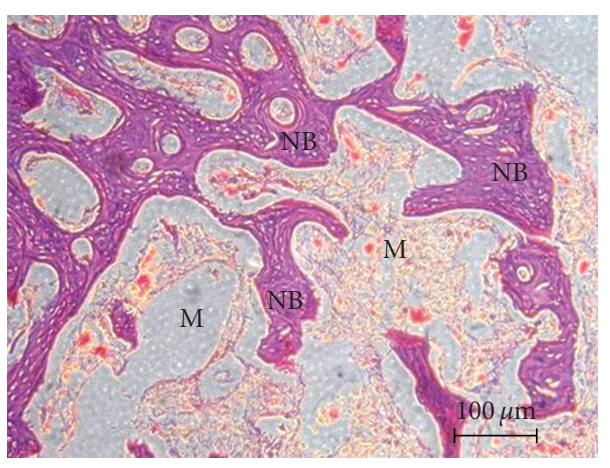

(b)

FIGURE 7: Implanted material powder in marrow cavity at 4 weeks in HAP group (a) and HAP/SF group $(b)(\times 100)$. New bone $(\mathrm{NB})$ began to form around the material $(\mathrm{M})$.

the HAP group. In the HAP/SF group, it can be seen that HAP/SF composite particles were adsorbed, and new bones were filled into the cavity. By comparison, it was also found that the new bone grew in both HAP/SF and HAP groups was

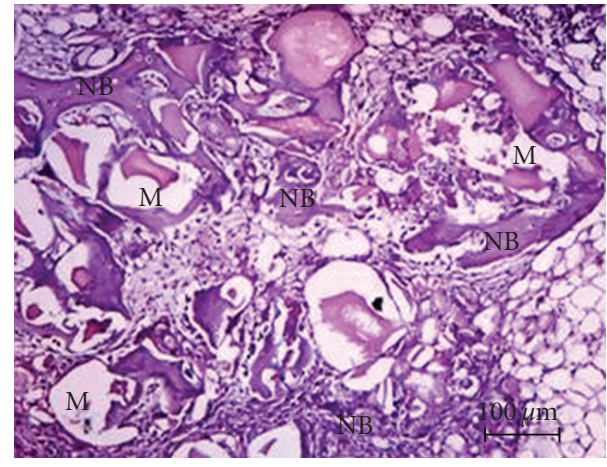

(a)

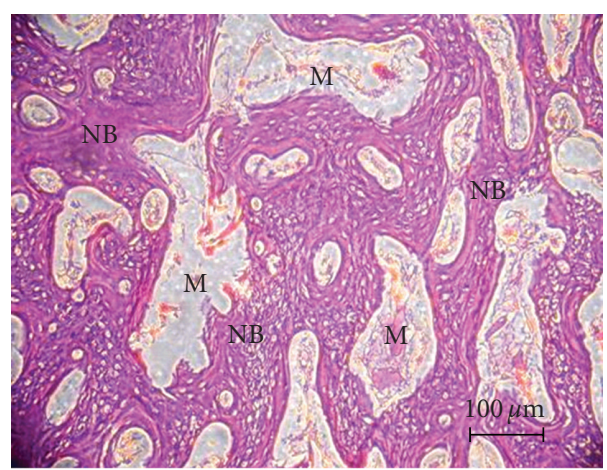

(b)

FIGURE 8: Implanted material powder in marrow cavity at 8 weeks in HAP group (a) and HAP/SF group $(b)(\times 100)$. The material $(M)$ was absorbed and new bone (NB) grew into the cavity.

a typical woven bone, but the bone in the former grew faster (Figure 7).

In the HAP group at 8 weeks, more HAP grains were adsorbed compared to that of 4 weeks, and more marrow tissue grew into the cavity. More new bone formed in the marrow tissue. In the HAP/SF group, the bone trabecula increased, became wider, and almost filled the cavity evenly (Figure 8).

At the beginning of new bone formation, the bone matrix, an organized network of collagen fibers, was found adherent to the HAP surface [26]. With little dissolution of HAP grains at 4 weeks [27], the new bone and collagen fibers enclosed the HAP grains. For the HAP/SF group, the new bone formed better at the beginning because of the good biocompatibility and bioactive of the composite [28]. The inflammatory responses to the composite initiated the degradation of the composite and the formation of new bone [29]. The degradation rate may be related to the weight of SF. Further study is needed to quantitatively evaluate the new bone formation and the rate of adsorption in vivo.

\section{Conclusions}

A novel HAP/SF bone-like composite biomaterial was developed by biomimetic synthesis. In composition and microstructure, the composite has the same characteristics 
like natural bone. The composite has excellent biocompatibility in vitro and in vivo, which suggests that the developed $\mathrm{HAP} / \mathrm{SF}$ composite has a potential for the application in the repair of bone defects and bone regeneration.

\section{Acknowledgment}

This paper was supported in part by the Programs for Science and Technology Development of Shaanxi province, China (Grant no. 200814k-03), the National Natural Science Foundation of China (Grant no. 30772440), Key Programs for Science and Technology Development of Anhui province, China (Grant no. 08010302196), and Stomatology College Youth Fund of Xi'an Jiaotong University.

\section{References}

[1] R. E. Unger, M. Wolf, K. Peters, A. Motta, C. Migliaresi, and C. J. Kirkpatrick, "Growth of human cells on a non-woven silk fibroin net: a potential for use in tissue engineering," Biomaterials, vol. 25, no. 6, pp. 1069-1075, 2004.

[2] T. Masuhiro, F. Giuliano, M. Norihiko, and A. Giulia, "Preparation and application of porous silk fibroin materials," Journal of Applied Polymer Science, vol. 54, no. 4, pp. 507-514, 1994.

[3] M. Santin, A. Motta, G. Freddi, and M. Cannas, "In vitro evaluation of the inflammatory potential of the silk fibroin," Journal of Biomedical Materials Research, vol. 46, no. 3, pp. 382-389, 1999.

[4] H.-J. Jin, J. Chen, V. Karageorgiou, G. H. Altman, and D. L. Kaplan, "Human bone marrow stromal cell responses on electrospun silk fibroin mats," Biomaterials, vol. 25, no. 6, pp. 1039-1047, 2004.

[5] C. Vepari and D. L. Kaplan, "Silk as a biomaterial," Progress in Polymer Science, vol. 32, no. 8-9, pp. 991-1007, 2007.

[6] C. Du, F. Z. Cui, W. Zhang, Q. L. Feng, X. D. Zhu, and K. De Groot, "Formation of calcium phosphate/collagen composites through mineralization of collagen matrix," Journal of Biomedical Materials Research, vol. 50, no. 4, pp. 518-527, 2000.

[7] M. Kikuchi, S. Itoh, S. Ichinose, K. Shinomiya, and J. Tanaka, "Self-organization mechanism in a bone-like hydroxyapatite/collagen nanocomposite synthesized in vitro and its biological reaction in vivo," Biomaterials, vol. 22, no. 13, pp. 1705-1711, 2001.

[8] L. Wang, R. Nemoto, and M. Senna, "Changes in microstructure and physico-chemical properties of hydroxyapatite-silk fibroin nanocomposite with varying silk fibroin content," Journal of the European Ceramic Society, vol. 24, no. 9, pp. 2707-2715, 2004.

[9] Q.-L. Li, Z.-Q. Chen, B. W. Darvell et al., "Biomimetic synthesis of the composites of hydroxyapatite and chitosanphosphorylated chitosan polyelectrolyte complex," Materials Letters, vol. 60, no. 29-30, pp. 3533-3536, 2006.

[10] S.-H. Rhee and J. Tanaka, "Self-assembly phenomenon of hydroxyapatite nanocrystals on chondroitin sulfate," Journal of Materials Science: Materials in Medicine, vol. 13, no. 6, pp. 597-600, 2002.

[11] T. Akari, O. Chikara, M. Toshiki, T. Hiromi, Y. Masao, and T. Masao, "Deposition of bone-like apatite on silk fiber in a solution that mimics extracellular fluid," Journal of Biomedical Materials Research Part A, vol. 65, no. 2, pp. 283-289, 2003.
[12] L. Wang, G.-L. Ning, and M. Senna, "Microstructure and gelation behavior of hydroxyapatite-based nanocomposite sol containing chemically modified silk fibroin," Colloids and Surfaces A, vol. 254, no. 1-3, pp. 159-164, 2005.

[13] X. D. Kong, F. Z. Cui, X. M. Wang, M. Zhang, and W. Zhang, "Silk fibroin regulated mineralization of hydroxyapatite nanocrystals," Journal of Crystal Growth, vol. 270, no. 1-2, pp. 197-202, 2004.

[14] M. C. Chang and J. Tanaka, "FT-IR study for hydroxyapatite/collagen nanocomposite cross-linked by glutaraldehyde," Biomaterials, vol. 23, no. 24, pp. 4811-4818, 2002.

[15] T. Masami and M. Takehisa, "Surface functional group dependence on apatite formation on self- assembled monolayers in a simulated body fluid," Journal of Biomedical Materials Research, vol. 34, no. 3, pp. 305-315, 1997.

[16] F. Tsutomu, T. Tetsushi, K. Akio, A. Mitsuru, and T. Yasushi, "Preparation and characterization of apatite deposited on silk fabric using an alternate soaking process," Journal of Biomedical Materials Research, vol. 50, no. 3, pp. 344-352, 2000.

[17] Y. Li, Y. Cai, X. Kong, and J. Yao, "Anisotropic growth of hydroxyapatite on the silk fibroin films," Applied Surface Science, vol. 255, no. 5, pp. 1681-1685, 2008.

[18] M. C. Chang, C.-C. Ko, and W. H. Douglas, "Conformational change of hydroxyapatite/gelatin nanocomposite by glutaraldehyde," Biomaterials, vol. 24, no. 18, pp. 3087-3094, 2003.

[19] D. D. Deligianni, N. Katsala, S. Ladas, D. Sotiropoulou, J. Amedee, and Y. F. Missirlis, "Effect of surface roughness of the titanium alloy Ti-6Al-4V on human bone marrow cell response and on protein adsorption," Biomaterials, vol. 22, no. 11, pp. 1241-1251, 2001.

[20] D. D. Deligianni, N. D. Katsala, P. G. Koutsoukos, and Y. F. Missirlis, "Effect of surface roughness of hydroxyapatite on human bone marrow cell adhesion, proliferation, differentiation and detachment strength," Biomaterials, vol. 22, no. 1, pp. 87-96, 2001.

[21] G. Xiao, D. Wang, M. D. Benson, G. Karsenty, and R. T. Franceschi, "Role of the $\alpha 2$-integrin in osteoblast-specific gene expression and activation of the Osf2 transcription factor," Journal of Biological Chemistry, vol. 273, no. 49, pp. 3298832994, 1998.

[22] Q.-L. Li, M.-Y. Wu, L.-L. Tang, J. Zhou, Y. Jiang, and B. W. Darvell, "Bioactivity of a novel nanocomposite of hydroxyapatite and chitosan-phosphorylated chitosan polyelectrolyte complex," Journal of Bioactive and Compatible Polymers, vol. 23, no. 6, pp. 520-531, 2008.

[23] Q.-L. Li, N. Huang, J. Chen, C. Chen, J. Chen, and H. Chen, "Endothelial cell and platelet behavior on titanium modified with a mutilayer of polyelectrolytes," Journal of Bioactive and Compatible Polymers, vol. 24, no. 2, pp. 129-150, 2009.

[24] N. Minoura, S.-I. Aiba, Y. Gotoh, M. Tsukada, and Y. Imai, "Attachment and growth of cultured fibroblast cells on silk protein matrices," Journal of Biomedical Materials Research, vol. 29, no. 10, pp. 1215-1221, 1995.

[25] K. Inouye, M. Kurokawa, S. Nishikawa, and M. Tsukada, "Use of Bombyx mori silk fibroin as a substratum for cultivation of animal cells," Journal of Biochemical and Biophysical Methods, vol. 37, no. 3, pp. 159-164, 1998.

[26] J. D. De Bruijn, C. A. Van Blitterswijk, and J. E. Davies, "Initial bone matrix formation at the hydroxyapatite interface in vivo," Journal of Biomedical Materials Research, vol. 29, no. 1, pp. 8999, 1995. 
[27] A. E. Porter, N. Patel, J. N. Skepper, S. M. Best, and W. Bonfield, "Comparison of in vivo dissolution processes in hydroxyapatite and silicon-substituted hydroxyapatite bioceramics," Biomaterials, vol. 24, no. 25, pp. 4609-4620, 2003.

[28] X. Jiang, J. Zhao, S. Wang et al., "Mandibular repair in rats with premineralized silk scaffolds and BMP-2-modified bMSCs," Biomaterials, vol. 30, no. 27, pp. 4522-4532, 2009.

[29] L. Meinel, S. Hofmann, V. Karageorgiou et al., "The inflammatory responses to silk films in vitro and in vivo," Biomaterials, vol. 26, no. 2, pp. 147-155, 2005. 

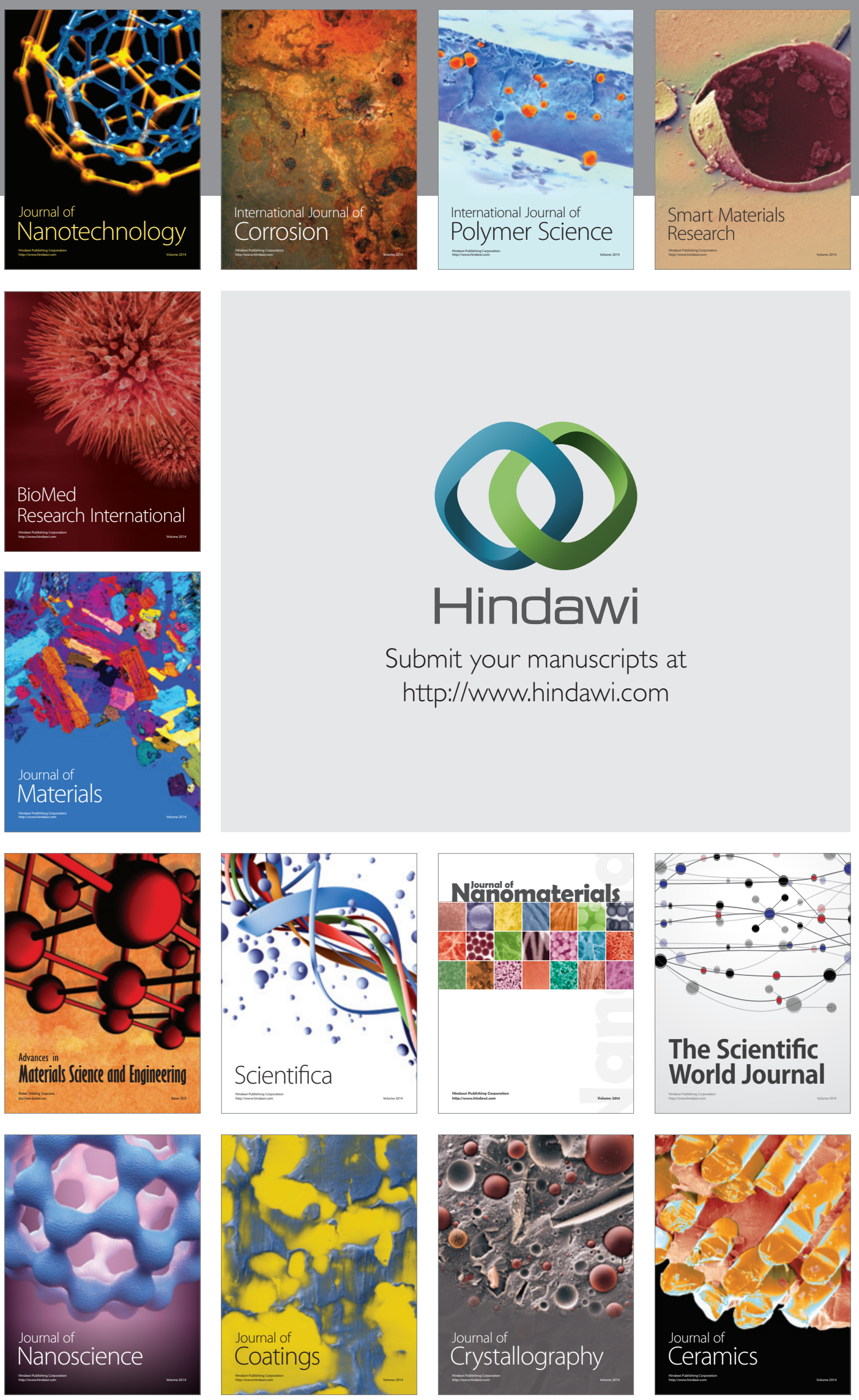

The Scientific World Journal

Submit your manuscripts at

http://www.hindawi.com

\section{World Journal}

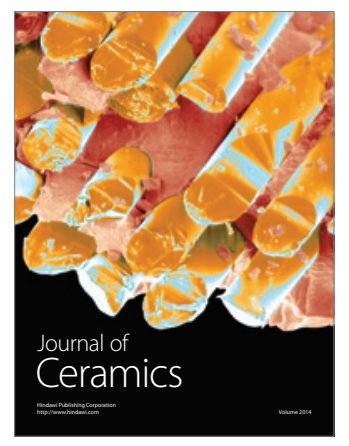

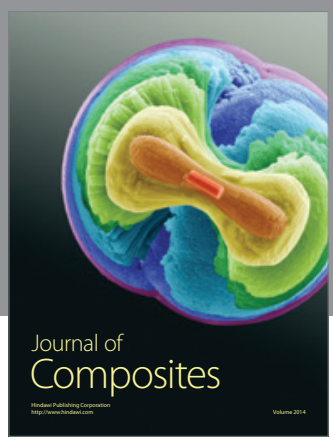
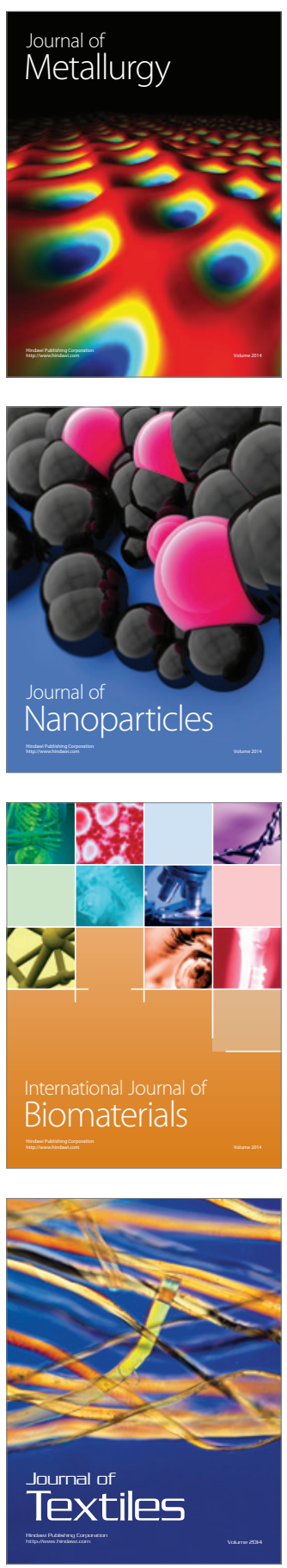